\title{
Analysis and Research on the Influence of University Discipline based on Incites and ESI
}

\section{_-Taking Materials Science of Jianghan University as an example}

\author{
Yan Ge \\ Library of Jianghan University, Wuhan, Hubei, 430056, China \\ geyan_zi@qq.com
}

Keywords: Discipline Construction; University; Incites; ESI; Academic Influence of Discipline

\begin{abstract}
At present, researchers have carried out many theoretical and empirical studies on the discipline analysis and evaluation based on ESI and Incites. The research on the research institutions and disciplines which don't belong to top 1\% worldwide ranking of ESI is relatively rare. This paper takes Materials Science of Jianghan University as an example to explore how to use Incites and ESI database through web of Science Documents, Times cited, Category Normalized Citation Impact to know the competitiveness and influence of it. According to the analysis, Materials Science is in the leading position in Jianghan University, but its influence is low outside the university. This paper is expected to provide a feasible approach to analyzing the academic influence for the research institutions and disciplinary fields which have not entered global top $1 \%$ of ESI.
\end{abstract}

\section{Introduction}

Scientific and reasonable evaluation of scientific research performance can provide a valuable reference basis for scientific research institutions to optimize resource allocation and the layout of disciplines [1]. With the development of bibliometrics, it has become a generally accepted method to measure the competitiveness and influence of scientific research in colleges and universities by the performance of scientific research achievements. In recent years, statistical analysis on the citation based on ESI, InCites and Web of Science has gradually attracted the attention of universities and the society[2]. More and more colleges and universities have set the number of the top $1 \%$ worldwide ranking of ESI as one of the development goals.

At present, researchers have carried out many theoretical and empirical studies on the subject analysis and evaluation based on ESI, Incites. The research results are mainly focused on the research institutions and discipline which belong to top 1\% worldwide ranking of ESI [3], while the research on the research institutions and disciplines which don't belong to top $1 \%$ worldwide ranking of ESI is relatively rare. Taking the material science of Jianghan University as an example, this paper discusses how to use Incites and ESI database to analyze, compare and evaluate the influence of university disciplines in order to provides a feasible way for the research institutions which don't belong to top $1 \%$ worldwide ranking of ESI.

\section{Data Sources}

Database introduction. The data in this paper come from InCites and ESI database. InCites and ESI database is a comprehensive scientific research evaluation and analysis platform designed and developed by Thomson Reuters Group in the United States. It is based on the data of Web of Science. It can track the research output and influence of colleges and universities, analyze the distribution of dominant disciplines, current situation and development trend of Scientific Research performance [4].

Data selection. Date of data acquisition is 10 September 2018. This paper selects materials science discipline, takes 2008 to 2018 as the analysis period, and adopts the ESI discipline classification model. The literature types are article and review。 
Analysis Indicators. The influence of discipline can be measured by web of Science Documents, Times Cited, and Category Normalized Citation Impact (hereinafter referred to as CNCI), \% International Collaborations, Citation Impact, etc.. Web of Science Documents reflect the scientific research productivity of the discipline. Times Cited and CNCI can reflect the scientific influence of the discipline [5]. CNCI is a valuable and unbiased indicator of influence which exclude the impact of the year of publication, research fields, and types of literature. If the CNCI value is greater than 1, the cited performance of this group of papers is higher than the global average. If the value is equal to 1 , the cited performance of this group of papers is equal to the global average [6].

\section{Analysis of material Science in Jianghan University}

The papers of Materials Science mainly come from the college of chemistry and environmental engineering in Jianghan University. The Department of polymer materials and engineering which was established in 2009 has a master's degree in materials science and engineering. The college also has the Ministry of Education key Laboratory of Optoelectronic Chemical Materials and Devices which is the first approved key Laboratory of the Ministry of Education within the province of Municipal affiliation university.

Trends in number of Papers. 122 papers are published by Jianghan University since 2008. The number of papers shows an obvious upward trend as shown in Fig. 1. Especially from 2014 to 2018, the number of papers was 99 which accounts for $81.15 \%$ of the total papers in the past ten years.

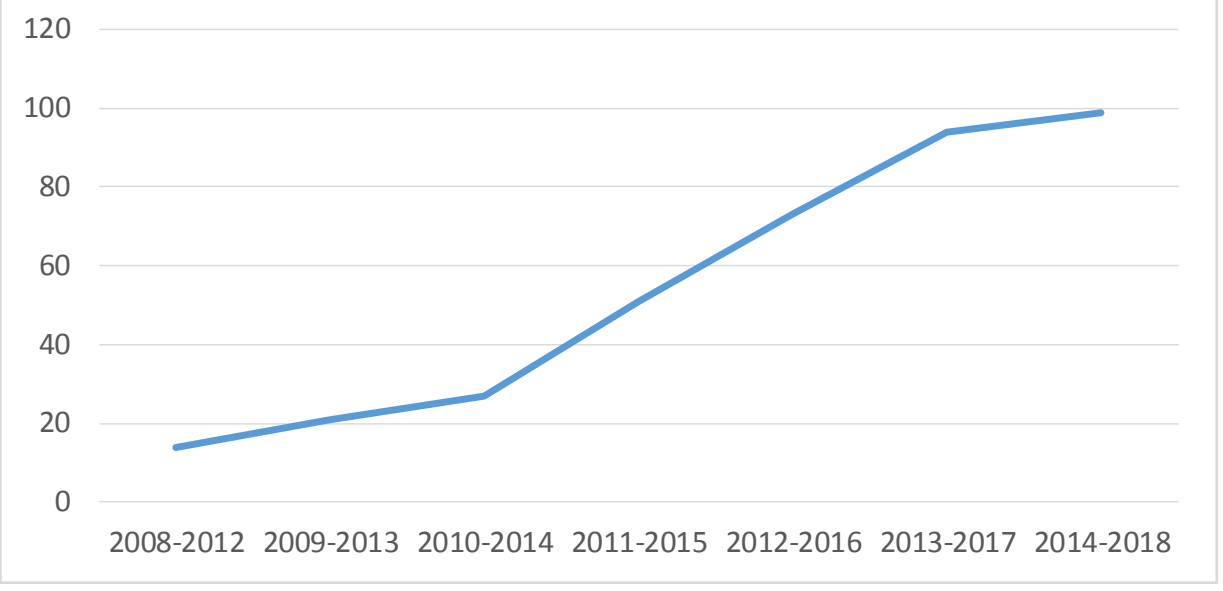

Figure 1. 5-Year Trend Graph

The ranking of Materials Science in Jianhan University. Material Science ranks second in all disciplines of Jianghan University in terms of web of Science Documents, Times Cited as shown in Table 1 . The CNCI value of Materials Science is 1.16 , which is slightly above the global average .

Table 1 Top 10 in all disciplines of Jianghan University

\begin{tabular}{lcccc}
\hline \multicolumn{1}{c}{ Rearch Field } & Rank & $\begin{array}{c}\text { Web of Science } \\
\text { Documents }\end{array}$ & CNCI & $\begin{array}{c}\text { Times } \\
\text { Cited }\end{array}$ \\
\hline Chemistry & 1 & 274 & 0.99 & 1840 \\
Materials Science & 2 & 122 & 1.16 & 1073 \\
Environment/Ecology & 3 & 109 & 1.4 & 862 \\
Clinical Medicine & 4 & 79 & 0.76 & 653 \\
Engineering & 5 & 73 & 0.7 & 417 \\
Physics & 6 & 82 & 0.54 & 410 \\
Biology \& Biochemistry & 7 & 84 & 0.48 & 379 \\
Plant \& Animal Science & 8 & 34 & 1.74 & 312 \\
\hline
\end{tabular}




\section{Comparative Analysis on the influence of material Science in 8 Universities}

In order to know the influence of material science of Jianghan University in recent ten years, this paper choose Hubei University, Ningbo University, Wuhan Institute of Tecology, Qingdao University, Three Gorges University, Yangtze University, Hubei University for Nationalities as the benchmark for comparison. A comparative analysis is made from web of Science Documents, Times Cited, CNCI , \% International Collaborations, Citation Impact, etc.. The Materials Science of Hubei University, Ningbo University, and Qingdao University have all been ranked among the top $1 \%$ of ESI .

Comparison of Web of Science Documents and the Times Cited. Hubei University, Ningbo University and Qingdao University have more published papers and cited times than the other universities. The ranking of Jianghan University is generally backward as shown in Fig. 2.

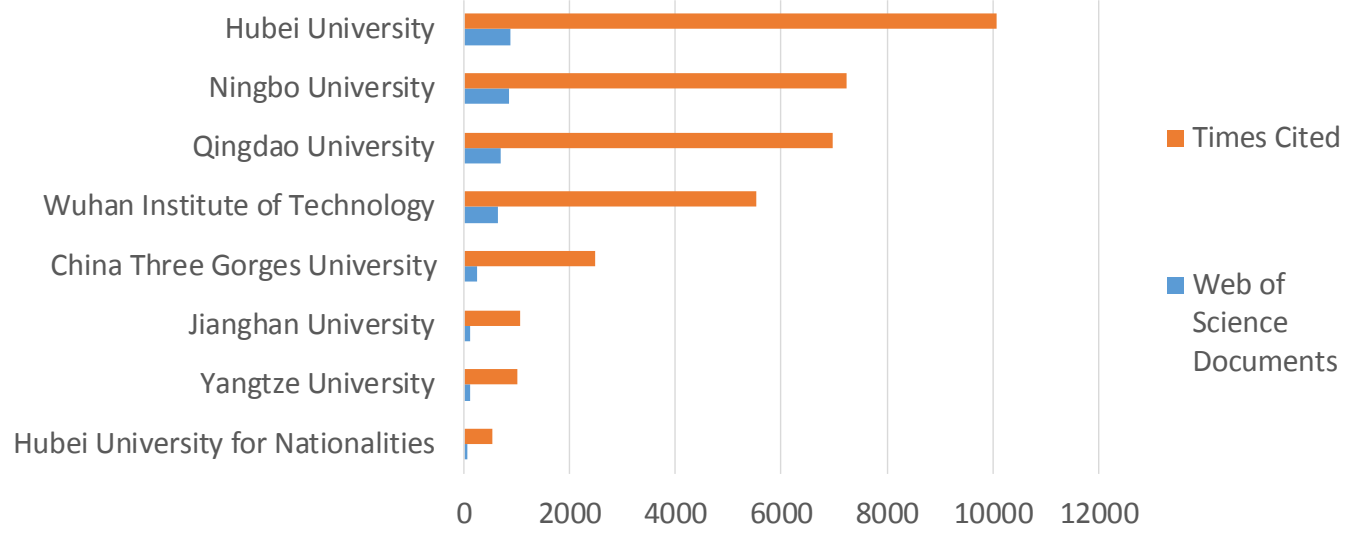

Figure 2. Comparison of Web of Science Documents and Times Cited

Comparative Analysis of International Cooperation. As shown in Fig. 3, proportion of papers on international cooperation of Qingdao University, Hubei University, and Ningbo University and Wuhan Institute of Technology are relatively high . Jianghan University ranked fourth, which is at a moderate level. There is a positive correlation between \% International Collaborations, CNCI and Citation Impact As shown in Fig. 4. Qingdao University and Hubei University which are among the top $1 \%$ institutions in ESI have high proportion of papers on International Cooperation. The proportion of international cooperation of Jianghan University is at the middle level, and the value of CNCI is slightly higher than the global average.

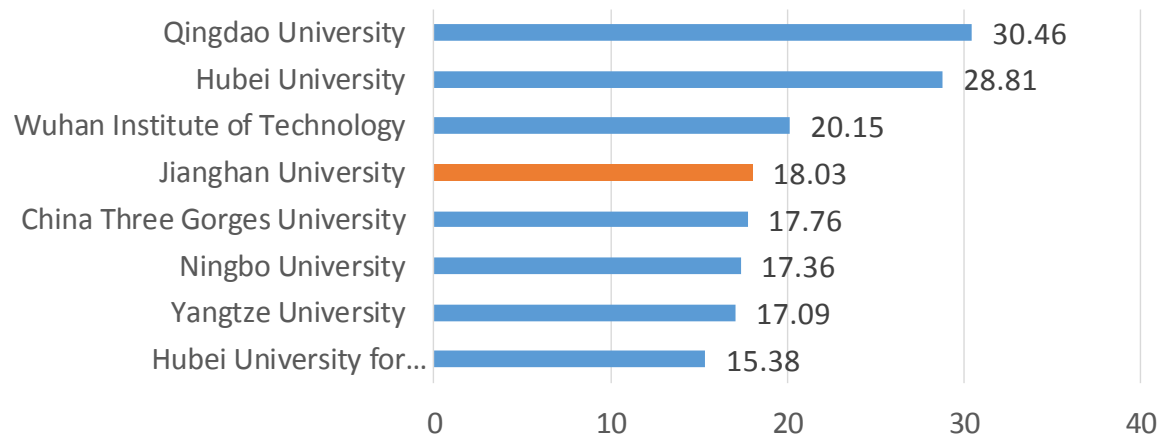

Figure 3. \% International Collaborations Contrast diagram 


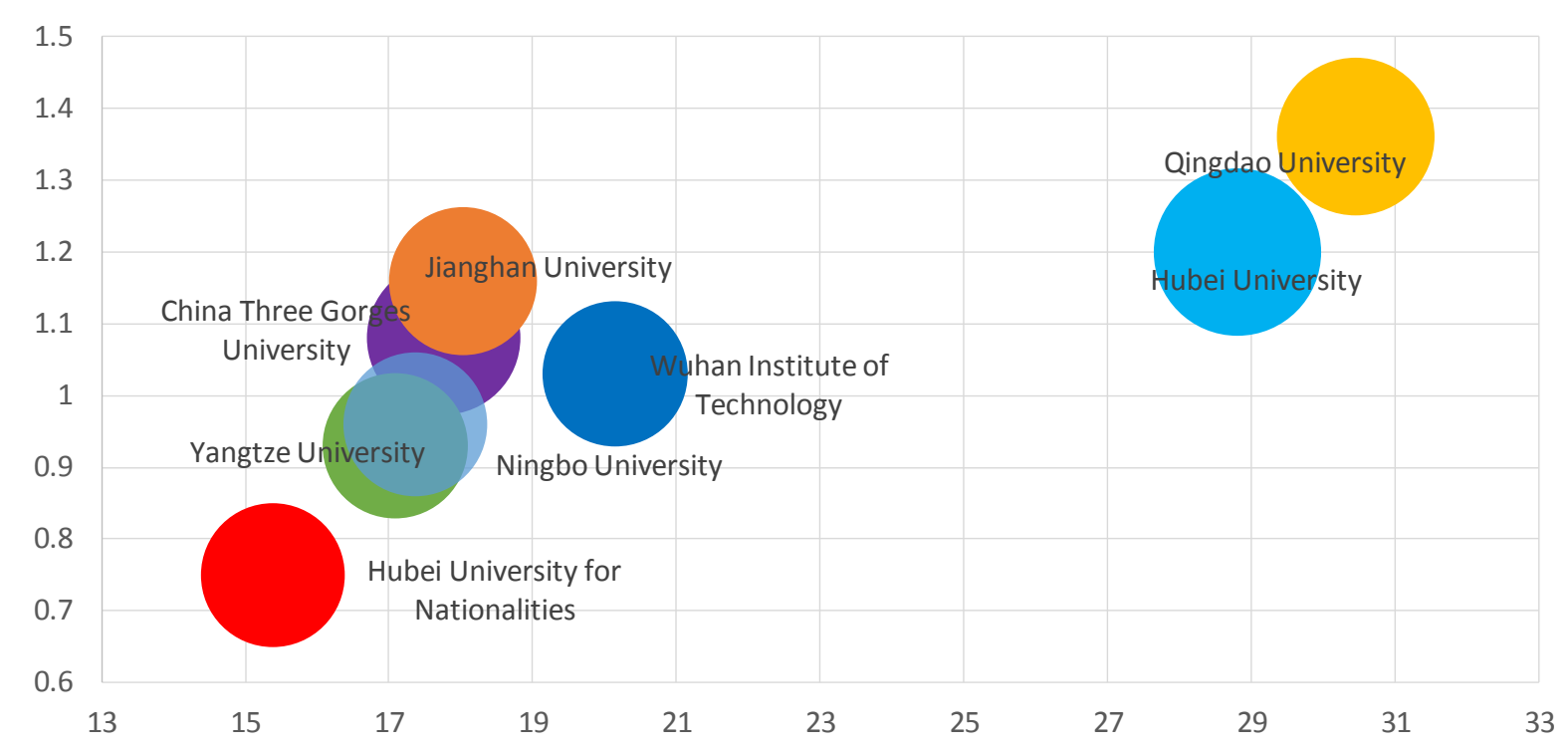

Figure 4. Relationship between International Collaborations and CNCI

Comparison of ESI Threshold. The threshold which can access top 1\% worldwide ranking of ESI is 5234 as shown in Fig. 5. The materials science of Hubei University, Ningbo University, and

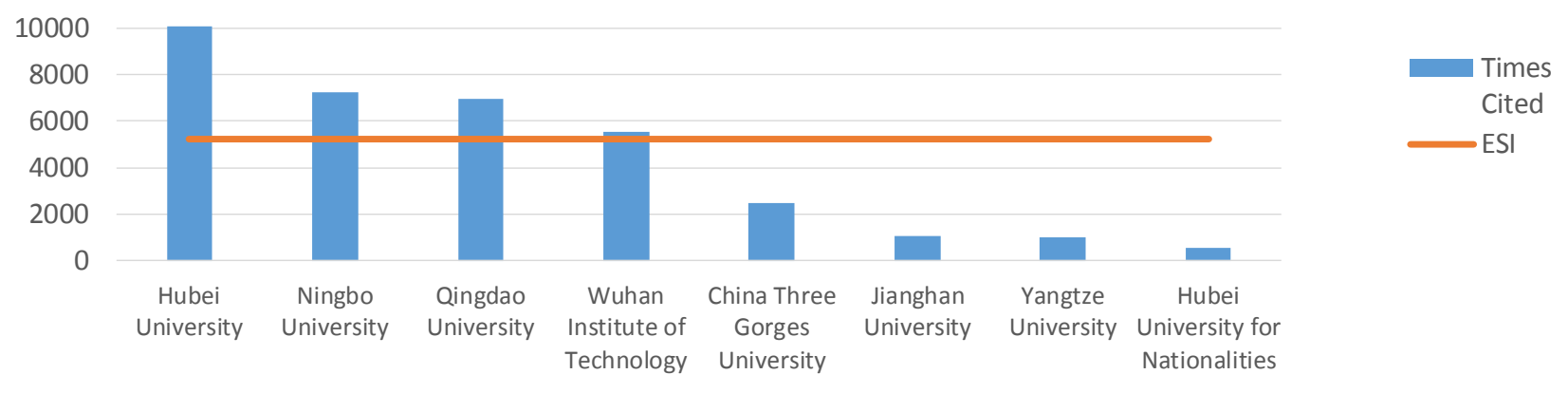

Figure 5. Comparison of ESI Threshold

Qingdao University have all been ranked among the top 1\% of ESI. Three Gorges University, Jianghan University, Yangtze University and Hubei Institute for Nationalities still have a long way to go before they can enter ESI.

\section{Conclusions}

The number of papers published by Jianghan University has increased significantly in the past 5 years. Judging from web of Science Documents, Times Cited and CNCI, the discipline of Materials Science is at the leading level in Jianghan University. The amount of papers issued in Materials Science has been greatly increased, which is closely related to This is related to Jianghan University's increased investment in the college and the college leaders' emphasis on discipline construction. The college has introduced high-level talents from home and abroad in recent years. The college also pays attention to international exchanges and cooperation, and has established good cooperative relations with the University of Western Ontario and the University of Alberta [8].

From the comparative analysis of the influence of Materials Science in 8 universities, we can see that the number of papers published by Jianghan University is small, cited frequency is low, and the quality of the papers and the proportion of papers on international cooperation are at the medium level. The influence of Materials Science of Jianghan University is low on the whole .

Jianghan University should further improve the evaluation mechanism of scientific research, encourage teachers and researchers to write multiple papers, and pay attention to improving the 
quality of papers. It is necessary to train and introduce a group of scientists who have a certain influence in the international academic circles of material science. Jianghan University also needs to strengthen cooperation with other institutions, especially international authorities, and should lay emphasis on intersection and integration of disciplines.

\section{References}

[1] X.L. Liu, S.Y. Zhang and S.S. Gai: Journal of Modern Information, Vol. 36 (2016) No.3, p. 172.

[2] L. Han, L.R. Guo and D.M. Kang: Information Research, Vol. 232 (2017) No.2, p27.

[3] L. Wang: Journal of Intelligence Vol. 36 (2017) No.2, p. 53.

[4] J. Wang: Journal of Hefei University of Technology (Social Science) Vol. 29 (2015) No.6, p. 124

[5] J.P. Qiu, R.Y. Zhao and R.M. Ma: Evaluation \& Management Vol. 5 (2007) No.1, p. 33.

[6] Information on https://wenku.baidu.com/view/3ebf8c47941 ea76e59fa0400.html?from=search.

[7] Information on http://hhxy.jhun.edu.cn/55/6a/c1264a87402/page.htm. 